Research Article

\title{
Professionals' Views on Offering Pre-Natal Testing for Adult Onset Cancer Susceptibility. Reconciling Personal and Professional Ethical Conflicts, Coping Strategies and Need for Policy Transparency
}

\author{
Lorraine Cowley $^{1,2,+, *}$, Mari Jones ${ }^{3,+}$
}

1. Newcastle upon Tyne NHS Foundation Hospitals Trust, Northern Genetics Service, Newcastle upon Tyne, United Kingdom; E-Mail: Icowley@nhs.net

2. PEALS (Policy Ethics and Life Sciences), Newcastle University, Newcastle upon Tyne, United Kingdom.

3. Liverpool Centre for Genomic Medicine, Liverpool Women's NHS Foundation Trust, Liverpool, United Kingdom; E-Mail: Mari.Jones@lwh.nhs.uk

+ These authors contributed equally to this work.

* Correspondence: Lorraine Cowley; E-Mail: Icowley@nhs.net

Academic Editor: Joep Geraedts

Special Issue: Genetic Testing

OBM Genetics

2020, volume 4, issue 2

doi:10.21926/obm.genet.2002108
Received: December 30, 2019

Accepted: April 20, 2020

Published: April 26, 2020

\begin{abstract}
This paper explores views of healthcare professionals (HCPs) in genetics and foetal medicine settings on offering pre-natal diagnosis (PND) for adult onset cancer susceptibility (AOCS) genes. Study participants came from different disciplinary backgrounds and all had considered implications of offering PND for AOCS, directly or indirectly, from professional and personal perspectives. Foetal medicine and genetics teams are accustomed to offering PND with the possibility of terminating an affected foetus on the basis of preventing severe physical or mental handicap to the child $[1,2]$. In this qualitative study, participants were invited to semi-structured interviews. Using a narrative approach, participants had space to follow their thought processes. Narratives were thematically analysed. Whilst participants
\end{abstract}

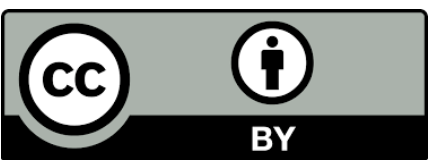

(C) 2020 by the author. This is an open access article distributed under the conditions of the Creative Commons by Attribution License, which permits unrestricted use, distribution, and reproduction in any medium or format, provided the original work is correctly cited. 
predominantly agreed that PND for AOCS does not fulfil Ground E for termination, they acknowledged difficulties in defining 'severe' taking into account patients' experiences and perspectives in the reproductive decision-making process. Findings indicated that many participants felt uneasy about the prospect of offering PND for a condition which does not, in their view, conform to the types of conditions typically seen and accepted in this context. Inconsistencies emerged around what participants think 'is' offered and what reproductive choices 'should' be offered to patients in this context. We discuss coping strategies used by HCPs to reconcile personal and professional ethical challenges. Offering Pre- implantation Genetic Testing (PGT) for AOCS is conflated with offering PND by some participants and we discuss possible reasons for this. We also explore potential impacts of PND for AOCS on mainstreaming cancer genetics. Our research demonstrates healthcare professionals' resilience in aiming to deliver compassionate care despite their personal and professional ethical conflicts. We argue that making policy about PND transparent and accessible will support professionals who feel conflicted about offering PND in new contexts such as AOCS genes.

\section{Keywords}

PGT; PND; ethics; adult-onset; cancer susceptibility; policy transparency; mainstreaming

\section{Introduction}

This paper discusses the views of health care professionals (HCPs) in genetics and foetal medicine settings working in a UK teaching hospital who were involved, directly or indirectly, with offering pre-natal testing for adult onset genetic susceptibility to cancer. The focus of the paper highlights HCPs reported experiences and explores possible reasons for feeling ethical conflicts over this issue. We discuss their coping strategies for dealing with personally challenging ethical conflicts and share their suggestions for ways to ameliorate conflicts.

\subsection{Usual Practices in the UK}

In Europe, and globally there is substantial variation in legal access to termination of pregnancies which influences reproductive medicine practices [3, 4]. However, health professionals working in contemporary foetal medicine and genetics settings in the UK are accustomed to offering PND and termination for conditions that present early in life; exhibit high to complete penetrance; and have limited effective treatments $[1,2]^{1}$. Following the Abortion Act (1967), to arrange termination, two doctors must agree the grounds for the procedure as set out on the HSA1 form (See Figure 1). Termination of affected pregnancies in foetal medicine usually fulfil Ground E criteria (the grounds for termination of a pregnancy in England and Wales, 2013) [5]. Terminating a foetus under the terms of Ground E is based on preventing severe physical or mental handicap to the child [1,2]. PND is additionally available at some UK centres for some

\footnotetext{
${ }^{1}$ In this paper, we refer to these conditions as defining an affected pregnancy.
} 
later-onset conditions such as Huntington's disease and adult susceptibility to breast and ovarian cancers (BRCA1 and BRCA2). This paper focuses only on adult onset cancer susceptibility (AOCS).

We hereby certify that we are of the opinion, formed in good faith, that in the case of... (Full name of pregnant woman and usual place of residents)...

A The continuance of the pregnancy would involve risk to the life of the pregnant woman greater than if the pregnancy were terminated;

B The termination is necessary to prevent grave permanent injury to the physical or mental health of the pregnant woman;

C The pregnancy has NOT exceeded its 24th week and that the continuance of the pregnancy would involve risk, greater than if the pregnancy were terminated, of injury to the physical or mental health of the pregnant woman;

D The pregnancy has NOT exceeded its 24th week and that the continuance of the pregnancy would involve risk, greater than if the pregnancy were terminated, of injury to the physical or mental health of any existing child(ren) of the family of the pregnant woman;

E There is a substantial risk that if the child were born it would suffer from such physical or mental abnormalities as to be seriously handicapped.

Figure 1 Excerpt from 'Form HSA1: grounds for carrying out an abortion' (2013).

\section{Planning a family}

Cancer risk genes can be passed on to any children you have. If your predictive genetic test is positive and you want to start a family, you have several options. You can:

- Have your children without any intervention, and risk your child inheriting the faulty gene

-Adopt a baby

- Use donor eggs or donor sperm (depending on who carries the faulty gene) to avoid passing on the faulty gene

-Discuss prenatal testing with your doctor. This test may be done in pregnancy and can tell you if your baby has the cancer gene

- Have pre-implantation genetic diagnosis - a technique used to select embryos that have not inherited the faulty gene. However, there is no guarantee this technique will result in a successful pregnancy, and funding for it on the NHS may not be available to all patients

Figure 2 Information about PND: part of 'Predictive genetic tests for cancer risk genes' NHS webpage (which refers to BRCA1 and BRCA2 as example cancer risk genes). NHS website (Choices NHS 2019) [6].

PND for BRCA1 and BRCA2 is publicised as being available as an option in pregnancies at risk on the NHS website [7] (See Figure 2) and other NHS publicly accessible documents (NHS Choices, The 
Royal Marsden NHS Trust 2016) [8]. The terms 'AOCS' and BRCA1, BRCA2 will be used interchangeably throughout the remaining text.

\subsection{Pre-Implantation Genetic Testing - an Alternative to PND}

Many patients with genetic conditions opt to have their children without intervention. For some who want intervention, termination of an affected pregnancy is not an option that they can or want to consider. Preimplantation Genetic Testing (PGT) ${ }^{2}$ is an alternative that avoids terminating a pregnancy for patients with some genetic conditions [9-11]. The regulations around PGT are variable across Europe [12] and controversially PGT is not regulated in the USA [13]. In the UK PGT is regulated by the Human Fertility and Embryology Association (HFEA) and it is offered through the NHS to prospective parents who meet strict personal health and social criteria (NHS England Clinical Reference Group for Medical Genetics 2014) [14]. PGT for BRCA1 was first approved by the HFEA on a case-by-case basis in 2007; the HFEA subsequently provided general approval to provide PGT for BRCA1 mutations in 2009 and for BRCA2 in 2010 [15]. HCPs offering PGT in the UK have expressed concerns about extending PGT to include late-onset, lower penetrance conditions, including AOCS [16]. Their concerns were about whether AOCS was a 'severe' enough condition to offer PGT, on the basis that cancer genes such as BRCA have reduced penetrance and cancer is a treatable condition [16]. A key debate in HCPs attitudes to all reproductive interventions, including PGT, is concerning what is considered a 'severe' condition [17]. One UK study reported that HCPs considered perceived forecast of a child's life expectancy in their decisions about offering PND [18]. In France survey research has highlighted that HCPs held contrasting views about whether offering PND and, or PGT for BRCA is 'acceptable' [19].

\subsection{Ethical Tensions}

If severity of a condition is a key factor in deciding when it is acceptable to offer reproductive interventions, it follows that having agreement on what is severe is important for HCPs in practice. In the UK HCP's attitudes to widening reproductive interventions were shown to be influenced by individual patients' perceptions of severity $[16,20]$. However, the same studies raised ethical questions about how, and whether, patient choice should have priority over possible societal consequences of those decisions. A possible societal consequence being the potential to stigmatise people with certain conditions if those conditions were screened-out prenatally [16, 20].

Whilst there is an expectation that HCP's are supportive of patients' reproductive decisions, sometimes it can be difficult to reconcile this with their professional frameworks. An example from a genetic counselling perspective is a scenario where prospective parents might choose to continue with a pregnancy following an unfavourable PND result for an adult-onset condition. In effect, such a scenario removes that child's right to make an independent informed decision

\footnotetext{
${ }^{2}$ PGT uses in vitro fertilisation (IVF) technology, whereby fertilised embryos are tested for the familial mutation of interest, and only unaffected embryos are implanted to the mother's womb [6].

In the UK, this is referred to as Pre implantation genetic diagnosis (PGD) and research participants of this study use this terminology. The authors use the broader term PGT in this context as in AOCS, identifying increased risk of a disease is distinct from making a diagnosis.
} 
around predictive testing for that condition and therefore is at odds with Genetics practitioners' professional frameworks [21, 22].

Previous studies have addressed the attitudes of patients with AOCS towards reproductive options [9-11]. Less is known about HCPs attitudes to PND for AOCS and in the UK research is especially limited [11]. A few studies have explored HCPs' views on reproductive options more generally, with some specifically addressing AOCS [16, 23].

These few studies have highlighted the complexities of developing a consensus around 'ethical principles' for PND and whether we should approach this in the same way as PGT. Our study specifically explores the views of Genetics and Foetal Medicine HCPs working in one UK NHS Foundation Trust on offering PND to women whose pregnancy is at fifty per cent risk of inheriting an AOCS gene.

\section{Methods}

We approached this subject with a qualitative interview study design. Qualitative semistructured interview methodology was chosen for this project because it is an exploration of a subject for which little is known and published [24].

\subsection{Sampling}

We interviewed 10 professionals from a UK NHS Foundation Trust. All were involved in offering a PND service but came from different disciplinary backgrounds including: Genetic Counsellor, Genetics Consultant, Clinical Scientist in Genetics, Foetal Medicine Consultant and Midwife. They all had direct or indirect involvement in discussions around, or offering, PND to patients/couples for AOCS. Whilst 15 professionals responded to the research invitation email, 10 participants were purposively selected based on representation of the professional groups and relevance of their experience in the area of PND. Participant recruitment was limited to 10 due to time and resource constraints of the project.

\subsection{Approach to Interview Methods}

Whilst everyone has a unique individual perspective based on their life experiences and views, we felt that specialism-specific views would be interesting to explore.

Face to face interviews were held in a suitable quiet meeting room at, or near, the participant's place of work, depending on their preference. An interview schedule was used highlighting indicative themes to explore. Using a narrative approach, the interviewer remained flexible, allowing the participants to follow their own thought processes, with opportunities to focus and expand on areas of their personal interest [25].

The interviewer was sensitive to possible emerging themes throughout the interview process, using constant comparative techniques to adapt questions and probes for subsequent interviews [26]. The researchers acknowledge that they and the participants have cultural and professional filters which influence the co-construction of narratives in qualitative interviews. By adopting a reflexive approach, the researchers recognised how their partiality may be transferred to the interview process and analysis [25]. Interviews lasted on average 46 minutes 30 seconds, were digitally audio-recorded, fully transcribed verbatim and anonymised. 


\subsection{Data Analysis}

The transcripts were thematically analysed using a voice-relational approach. In this approach, transcripts are read multiple times, each reading focusing on different voices and perspectives [27, 28]. This approach initially generated data about participants' views on PND for AOCS. In subsequent transcript readings, focusing on the emotion expressed by participants, themes were highlighted that captured areas where participants appeared to struggle to reconcile their personal and professional ethics. Narrative approaches gave participants space to articulate and potentially reconcile their internal conflicts.

Following analysis of the data, participants were presented with anonymised study findings via NHS email, with the opportunity for them to feedback on the data interpretation, known as respondent validation [29]. This approach helps to avoid misrepresentation of participants' views by asking research participants whether they feel the themes presented and data interpretation adequately reflects their perspective. No participants expressed unfavourable feedback on the data interpretation. Participants are identified in the data by profession and a number, for example, Genetic Counsellor 1. Numbers in each professional group are not published and some identifying details within quotes have been altered to protect participants' anonymity.

\subsection{Ethics Statement}

The Research and Development office at the NHS Trust confirmed their approval of this study, agreeing to act as sponsor. The Health Research Authority granted further approval. A separate favourable ethical opinion was obtained via the University Research Ethics Committee (proportionate review) at The University of Manchester.

\section{Findings}

In discussing their feelings about PND for AOCS, four overarching themes were identified: 'acceptance versus unacceptance of offering PND in the context of AOCS'; 'subjectivity of whether AOCS was severe enough a condition to justify PND'; 'gatekeeping of access to PND in this context'; and 'pushing the boundaries of what is acceptable grounds to offer PND'. The overall themes and subthemes are illustrated in Figure 3. We will briefly discuss overall findings but focus specifically on how HCPs cope with ethical conflicts and explore possible reasons for those conflicts.

Participants $(n=9 / 10)$ recalled that their initial reactions to the prospect of offering PND for AOCS were negative. Participants expressed: surprise, shock, questioned it, felt confusion, uneasiness, and if their roles permitted, had a slightly greater reluctance to get involved with such a request. After having time to adapt to the information, discussing with other HCPs, attending educational sessions in genetics and participating in this interview study, half of the participants did not express changes in their views, continuing to feel uncomfortable or uneasy about the prospect of offering this service. The following sections discuss how they coped with that. 


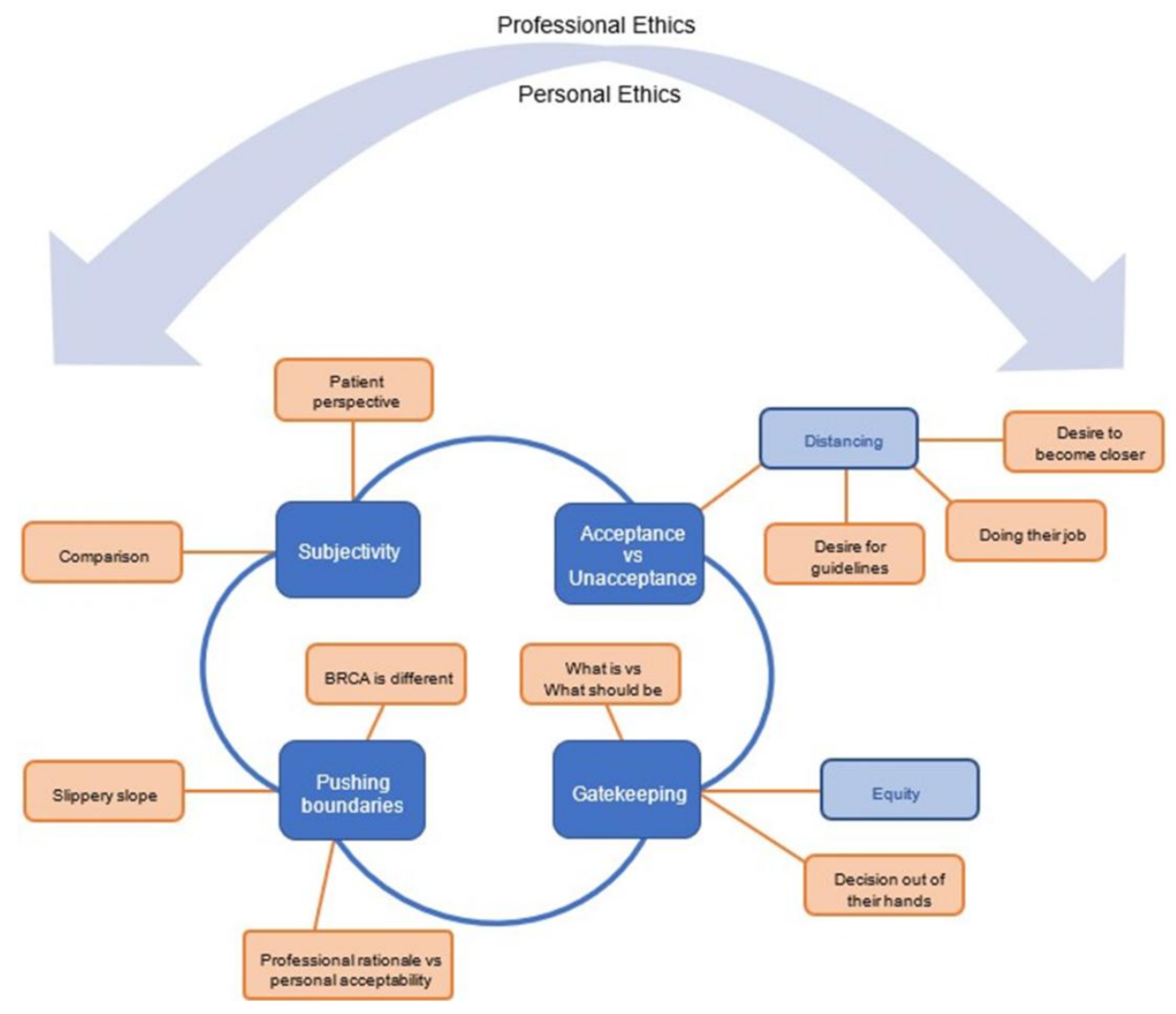

Figure 3 Personal and Professional Ethics - pre-natal testing for adult onset cancer susceptibility. Visual summary of themes.

\subsection{Coping Strategies}

Although the majority of participants had held or currently hold personal ethical reservations about offering PND for AOCS, they felt that it was part of their professional role to offer the service. Whether this was by choice (because they were able to reconcile their reservations), or by default (because they felt that it was a publicised service on NHS websites, see Figure 2). Their coping strategies in trying to reconcile their professional and personal stances on PND for AOCS drew on their emotional, psychological, policy information seeking and practical resourcefulness. Emotional coping strategies included patient empathy and compassion; psychological strategies included comparative analysis between PND and PGD, compartmentalising and self-denial; practical resourcefulness included co-counselling and avoidance. Participants' efforts at reconciling their feelings of ethical conflict illustrated their desire and need to act ethically. Following Kleinman's work on living a moral life [30] and Plummer's approach to narrative as 'stories we live by' [31], their efforts defined them as moral actors. Self-identifying as living a moral life is an important construct for personal well-being [32].

\subsubsection{Emotional Strategies -Empathy and Compassion}

All participants, whether working in a patient-facing role or not, and whether they personally agreed with it or not, expressed empathy and compassion about patients seeking PND for AOCS. They gave nuanced compassionate views of their feelings towards patients in this situation: 
Genetics Consultant 1: For me...it's kind of always tied up with the particular family and their reason for asking for prenatal...so...umm (pause). I suppose that I feel...that it's not something that I would ever ask for, umm, but that when people do ask for that...there is usually something about their personal circumstances that makes that...umm, something that they would consider because actually most people wouldn't consider that. So if somebody does ask for that it would always make me think ok, what has happened to this person, why is this person asking for that as opposed to, this is kind of, you know...morally reprehensible, or... whatever.

The trigger for this participant's compassion and empathy is the request to have PND for AOCS. In the participant's view this is such an unusual request, that, in the view of the participant, the patient must have, what they consider are, good reasons to request it. It would be interesting to see how and whether the participant's views changed if their experiences of PND for AOCS were more commonplace and not an unusual practice. Participants also demonstrated compassion by showing appreciation of how subjective it is to define what is severe:

Genetics Consultant 2: In a family where many people have died as a result of the condition, then actually maybe you can justify prenatal diagnosis... for that family, this is a severe disease. So, the definition of severity isn't just a medical one it's actually got something to do with the perception of a family.

Empathy and compassion were coping strategies because they helped HCPs discursively manage their equivocal feelings about PND here. For the participants of this study, PND for BRCA1 and BRCA2 was offered in shared understandings of presumed contexts. As highlighted by this quote, participants presumed that patients requesting PND for AOCS have a significant family history of cancer. Making that assumption helped participants to imagine possible traumas this might evoke for a patient, thereby eliciting compassion and empathy. However, as genetic testing becomes mainstream, BRCA1 and BRCA2 testing will be widely offered in the diagnostic setting and mutations in BRCA may be identified in people who have cancer without having a family history of cancer. This will change the context of offering PND for AOCS. Whether it would weaken feelings of empathy and compassion from HCPs who feel conflicted about offering these services is unknown.

\subsubsection{Psychological Coping Strategies}

Participants who struggled to reconcile their personal ethical approach to PND for AOCS sometimes compartmentalised or denied their feelings about it in order that they could do their job and care for the patient.

A couple of participants alluded to their tendency to disassociate from their personal views and emotions as a coping strategy when dealing with difficult cases. Sometimes disassociation was more nuanced as illustrated when HCPs compared provision of PGD with PND. There was an assumption among some participants that if a genetic test is offered for PGD then it should be offered for PND. Whilst participants' narratives suggest inconsistencies around what participants think 'is' offered and what 'should' be offered to patients in context of reproductive choice, for some, comparison between PGD and PND was a coping strategy. It was used as a coping strategy 
because participants were aware that the HFEA had considered the ethics of PGD before granting the license. They interpreted this as a 'by proxy' ethical endorsement of PND:

Interviewer: Do you think ethics has an influence in sort of PGD versus the PND argument - personally or professionally? Or which one do you think influences you the most in that context?

Clinical Scientist 1: it just seems like a no brainer, why would you offer one without the other because it's the same thing in my eyes. Well if PGD's offered, why should prenatal diagnosis not be offered, because the two kind of go hand in hand. And just because someone might not be eligible for PGD, they should still be able to have that decision making process.

It is interesting that the term 'no-brainer' was used here. The same term 'no brainer' came up in findings from a previous study. Although not about PND, the previous study highlighted how an extended family with an inherited AOCS, Lynch Syndrome, brokered ethical decision-making about genetic testing. Those who had accepted a test viewed having a genetic test as a moral imperative. In test-accepters' views, those who declined a test had a right to choose, but had chosen wrongly. The term 'no brainer' was used in that context by several participants about the theoretical 'choice' to be tested, but some participants did not engage with forming an argument about choosing to have a genetic test since it appeared to them to be beyond choice and incomprehensible to decline, a 'no brainer' [31]. In the context of our study, the term, 'no brainer' could be interpreted as disassociation from the ethical questions of PGD and PND and perhaps suggests a desire for certainty about guidance. In addition to assuming that ethical consideration of PGD was a by proxy ethical evaluation of PND for AOCS, some participants relied on their clinical colleagues to have made an ethically justified judgment before making a request for PND. The following quote demonstrates this point:

Clinical Scientist 1: ... if we had a referral from our genetics team or clinical genetics team elsewhere, then I think we're, we would be entitled to assume that the proper counselling process had been gone through... and this decision had been arrived at as a result of that process ...after kind of hopefully a sort of general agreement, that, that was the best way for that particular family to go in those particular circumstances.

This is not a blind assumption, the participant would question decisions they felt disagreement about, but the assumption is based on respect for the professionalism and critical skills of their clinical colleagues.

In addition to comparing PGD to PND, participants discussed the underpinning grounds for termination of pregnancy to rationalise and reconcile potential ethical discordance. For example, participants alluded to the availability of termination for what they termed 'social' reasons, Ground C of the HSA1 form (See Figure 1)

The following quote exemplifies how participants referred to Ground $C$ for terminating a pregnancy to support the assumption that offering PGD for AOCS equates to offering PND for AOCS. The assumption avoids the issue that PND is usually only offered for foetal medical not social reasons. At this study site, in the context of AOCS termination would be offered based on Ground C, which is not the usual foetal medicine practice.

Genetics Consultant 1: I think, if a couple feel strongly enough that they want to end a pregnancy if it's affected, I'm not sure how that's kind of different from 
actually feeling that you can't continue a pregnancy at 11 weeks because of, you know social circumstances of what, for whatever reason. So, I think that it doesn't really make sense for things to say, no we're not going to do prenatal if somebody asks for it if you can have PGD for it.

Therefore, in the view of this participant, PND for AOCS is acceptable because Ground C is available and in that context, having any medical reason would be additional justification for PND. However, this rationale does not consider the ethical sensitivities of those who provide termination of pregnancy under Ground E and feel conflicted about PND for AOCS. In this study, difficulties for foetal medicine doctors to sanction termination for social reasons were highlighted because it was out of their usual sphere of practice. In principle, it was difficult for HCPs to disagree with PND for AOCS but in practice, it was problematic and troubling to carry out.

Whilst some used the comparison of PND and PGD for AOCS as a 'by proxy' ethical endorsement, and therefore a coping strategy, others disagreed with this.

Genetic Counsellor 2: ...it ties in because PGD became available for umm for those (BRCA1 and BRCA2) and I felt uncomfortable about that and then unfortunately you've then got that slip in about prenatal diagnosis as well..., PGD was almost the precursor ... And up until then, maybe prenatal diagnosis hadn't been, certainly hadn't been requested, because with that whole idea of termination and it was, there wasn't an alternative, and the termination bit was almost too, too difficult to think about. But when you had PGD in place, it almost made the prenatal diagnosis more palatable...

Although not used as a coping strategy, in this instance, this participant compares PGD and PND and views the arguments and ethical considerations about either as having become conflated.

In general, many participants' felt uneasy about the prospect of offering PND for a condition which does not, in their view, conform to the 'types' of conditions typically seen and 'accepted' and for which termination of pregnancy could be offered under Ground $\mathrm{E}$ of the abortion act.

Clinical Scientist 2: ... I still think that prenatal testing for a late onset condition should be quite, it should be near the bottom of the list... and also you know, I suppose (cancer) onset can be sort of 20s and 30s but where are we going to be technologically 20-30 years from now? I just think you've got to be more optimistic about things like later onset cancers, even if they are... early in life. But 20 years from now you just can't (know about future treatments) (considers and sighs) yeah, I think that's the difference.

It is interesting that this scientist's forward-looking approach is of optimism and confidence in the development of technology to treat cancer, but making this argument makes it more difficult to come to terms with feelings of ethical discordance about current practice of offering PND for AOCS.

\subsubsection{Practical Resourcefulness}

Practical resourcefulness was dependant on what was available to the professional group. One Genetic Counsellor had the resource to offer co-counselling. Co- counselling is an established approach in Genetic Counselling, practiced variously throughout England and Wales but generally in decline due to limited genetic counselling resource in services where there is an increase in 
genetic counselling demand. Within a genetic co-counselling session, two HCPs (genetic counsellor and another HCP) counsel patients with pre-agreed roles.

Genetic Counsellor 1. ... if I've felt that I was getting into the scenario that, in that case, I would then arrange a co-counselling session. And then if the couple said, we definitely want to go ahead with this...then that's their choice. I would then feel comfortable, that I had given them all the information, and not been judgemental or directive.

Here the participant reflexively acknowledges that they may find the situation personally, emotionally or ethically problematic. They suggest that the defined role of the co-counsellor is to be alert to their directiveness and moral judgment of the situation. This HCP is being reflexive about their own values and feelings, acknowledging that those feelings may have an impact on their professional practice.

In this data, there were differences between participants from differing disciplines in their approach to how responsible they might feel about participating in PND for AOCS such as BRCA if their personal ethical stance was troubled by that. Distancing was a strategy discussed by nonpatient facing HCPs. When asked how they felt about first hearing that PND was available for AOCS this non-patient facing participant responded:

Clinical Scientist 1: ...probably my personal feeling would (be) slightly greater reluctance to be involved in something that was late onset, and potentially treatable, so where there's potentially interventions for something like BRCA1 or BRCA2 mutations,... it's not $100 \%$ penetrant, umm,... if you're having a prenatal on the basis of termination for a bad result...umm, would that individual have felt that their life wouldn't be worth living with the prospect of breast cancer as opposed to not having a chance to be, to exist at all...

In this data, the possibility of avoidance of being involved in a situation where PND was offered for AOCS was only mooted if professionally possible, for example, only the non-patient facing participants might consider this as a strategy. Other HCPs working in genetics, clinical and laboratory, and foetal medicine units may well avoid situations as has been discussed in other areas of abortion care. One research group, for example, discuss how staff involved in abortions made distinctions about technical, material, temporal and occupational aspects of the work to demarcate a caregiver's role in an abortion [34]. Moving the boundaries of their involvement and categorising their role as being instrumental in the act of the abortion or reframing their role as supportive care after the event helped staff to create moral distance and reduce their sense of participation. In the same paper when staff had competing imperatives they prioritised professional obligations to patients [34], as did our respondents.

\subsubsection{Policy Information Seeking}

A key troubling area for HCPs who reported feeling discordance between their personal and professional ethics about PND for AOCS was the lack of transparent ethical or regulatory debate around this issue.

Participants' responses as to what might help in their struggle to reconcile their sense of ethical conflict are highlighted here: 
Foetal Medicine Consultant 1: ... I guess some open and transparent process for establishing a reasonable societal view, umm probably would help... but I think, umm I think part of the unease, does come from the fact that umm...it's an awareness that decisions are being made...that aren't black and white, ...but if we are a grown up mature...umm society that works together as a society rather than just lots of individuals, umm then I think some kind of system for saying that there is a reasonable body of opinion that would say that this is a reasonable thing to do...umm I think actually probably, would be helpful.

Foetal Medicine Consultant 2: Umm, and I'm not sure there's much discussion about regulation and about counselling and structure to that, and about what fits where from a legal framework. It just doesn't feel as if that conversation is, well it might be happening but if it's happening I'm not sure that we're aware of it very high on the agenda... and we certainly don't feel involved in that conversation.

Although desirable from most participants' perspectives, there was no explanation available to professionals for how PND was offered for AOCS and how it became part of the information on the NHS website. As Ormondroyd et al. (2011) [11] observe, there is no clear national agreement on whether PND should be considered equal to PGD, and earlier publications from the HFEA stated: 'Indications for the use of PGD are expected to be consistent with current practice in the use of (post-implantation) prenatal diagnosis (PND).'

However, this statement is not included in recent editions of the HFEA Code of Practice [11]. Addressing this lack of transparency but HCPs' apparent need to engage with the decision-making process, the authors have subsequently emailed the following: Subject Matter Expert (SME) within the NHS website, Public Health Department, NHS Screening Services England, NHS England and NHS Improvement. None of these departments have been able to explain the process of how PND for BRCA1 and BRCA2 was made available and publicised on the NHS website.

If some of the study participants presumed that when a test was deemed eligible for PGD licensing by the HFEA it was also available for PND then, perhaps, this was an influencing factor for curators of the NHS website. However, it is not the intention of the HFEA to consider the suitability of a genetic test, ethically or otherwise, for PND. Relating only to the context of PGD, the Standing Orders of the HFEA state: 'In particular, the panel must be satisfied that there is a significant risk that a person with the abnormality will have or develop a serious physical or mental disability, a serious illness or any other serious medical condition' [35].

Whereas the usual terms under which termination of pregnancy are offered following a bad news PND result state that it is offered when: '... there is a substantial risk that, if the child were born, it would suffer from such physical or mental abnormalities as to be seriously handicapped' (Ground E on the Abortion Notification Form DOH 2013) [2].

Although, according to the HCPs in this study, termination of pregnancy following PND for BRCA would not be justified under Ground $E$, it may be offered under Ground ' $C$ '. Whilst right of access to PGD may have been conflated with right of access to PND, the substantive basis under which each are operationalised, PGD being sanctioned to prevent illness in a person, PND to prevent illness in a child, are subtly yet significantly different. Conflating the terms and assuming that PGD is equivalent to PND is, in part, what has troubled HCPs ethical approaches to PND for AOCS in this study. Following up on this theme, the authors have also contacted the HFEA to inform them of this slippage in presumed endorsement of PND via PGD; their scientific policy officer responded 
indicating that PND is not within the HFEA's policy remit and they do not have any ongoing policy analysis in this area. They iterated that the HFEA license and monitor clinics carrying out IVF, donor insemination and human embryo research (HFEA email). To our knowledge, offering PND in the new context of AOCS is not regulated by any additional legal or professional framework other than existing laws set out in the Abortion Act.

\section{Discussion}

The provision of termination is one recognised area of medical practice where personal views may not be reconciled with professional practice [36-41]. Previous research has highlighted that where HCPs have felt conflicted they have discursively navigated their participation in termination of pregnancy care in order to reconcile their differences, sometimes referred to as moral work [42-44].

In this study, it is not the provision of termination that was problematic for participants, they work within a service that offers termination, but the changing context of providing termination of pregnancy that was troubling to HCPs. New technologies in healthcare are usually scrutinised prior to implementation, by legal and ethical processes. Publics are sometimes alerted to groundbreaking or potentially contentious technologies by the media, stimulating public discussion and drawing attention to a range of opinions [45]. Where new technologies are considered potentially ethically contentious there are forums for debate and they are duly considered before being implemented [45]. In the area of obstetric care and reproduction, we have highlighted the role of the HFEA in licensing the use of new technologies in reproductive settings such as PGT. However, the same robust consideration, or public scrutiny is not transparently evident when established reproductive technologies like PND are introduced for new conditions such as for a known inherited AOCS. From an international perspective, the variability in regulation of reproductive medicine makes it difficult to extrapolate how services might look if PGT was only offered for conditions where PND is offered. In this UK study these issues have arisen for participants where there is regulation for PGT, from an international perspective HCPs may face different complex challenges considering the variability in regulation of foetal medicine practices.

\subsection{Potential Mainstreaming Implications}

Participants in this study, as discussed within previous studies $[16,17,20]$, struggled with the concept of what conditions were severe enough to offer PND; with AOCS being problematic on the grounds of cancer being a treatable condition [46] and specifically BRCA genes being incompletely penetrant. It is interesting to consider then how the mainstreaming genetics agenda [47] may have an impact on these already present problems. With the introduction of mainstreaming genetic testing in the UK, genetic testing for disease susceptibility will increasingly become part of the diagnostic pathway in common diseases such as cancer. We see two possible issues that may affect the ethical debates about PND for AOCS. First, as mainstreaming genetic testing in the cancer setting is undertaken, more people will have BRCA mutations identified who do not have a strong, or perhaps any family history of cancer. Statistical analysis of this population may confound the current penetrance figures for BRCA further. We may be less certain what it means to have a BRCA mutation and therefore we may be less certain of the grounds to determine what is 'severe enough' before offering BRCA testing for PND. Secondly, the treatment of cancers is 
being driven by genetic analysis and BRCA status is being used, in some circumstances, to personalise medicine with the intention of increasing potential for cure $[48,49]$. It is paradoxical therefore that in one healthcare setting (Foetal medicine) a positive BRCA test result can mean termination of pregnancy whilst in the Oncology setting a positive BRCA result may signal better prognosis and treatment. Again, this argument will weaken further the grounds for offering PND with termination of pregnancy under Ground $E$ because cancer in a BRCA carrier may be viewed as being potentially more treatable. HCPs in this study reported that termination of pregnancy following PND for AOCS could still be offered under Ground ' $C$ '. In the context where improved treatment may be available and the empirical risk of developing cancer is potentially reduced, offering termination on the grounds of social reasons may influence the ethical landscape of reproductive medicine for HCPs caring for patients with AOCS genes.

\section{Conclusion}

Although not generalizable, this exploratory qualitative study has reported on coping strategies of 10 HCPs who have ethical conflicts about offering PND for AOCS. Regardless of their personal views on termination (not asked for) participants of our study coped by remaining professional and took pains to reconcile their feelings of disquiet so as not to detract from patient care. All were prepared to shelve their personal views to give the best and sensitive care to patients. All showed a great deal of empathy to the patient's position whether they agreed with offering this service or not and their professionalism and commitment to patients was apparent. They exhibited humility in decision-making; it was not about their perception but about the patient's. Participants vocalised acceptance that they were not in a position to judge another's motives for having PND. When conflicts were too great, participants resorted to compartmentalising their thoughts to enable them to continue to act professionally. They relied on colleagues to support them in times of ethical difficulty, and one genetic counsellor requested co-counselling as a means of operationalising support.

According to our findings, some HCPs assumed that offering PGT equates to offering PND for AOCS. Although there is no information available that was accessible to the authors about how PND for AOCS became available, it is possible that the same assumption was made at an NHS policy level, resulting in the availability of PND for BRCA being published on the NHS website. We cannot confirm this because of lack of transparent communication from policy makers. The grounds on which PGT is licensed for BRCA are significantly different to the grounds of offering termination following PND. In our study, this conflation of understanding led to a significant proportion of personal and professional ethical dilemmas in offering PND for AOCS. Whilst there were additional reasons why our participants reported feeling troubled by the practice of offering PND for AOCS, they were unanimous in their voicing that they wanted to offer the best care to patients in this situation.

Given that participants of our small exploratory study reported personal ethical conflicts about AOCS and PND, the authors argue for further research into this area and for transparency in policy making here. If policy on offering PND were to be transparent, whilst participants may have still experienced personal ethical conflict, they would also likely feel supported by the ethical and legal governance framework. It is this element which is asked for but which is missing from their coping strategies. Transparency of policy-making is what they needed and whilst that is available for PGT, 
it is not available for PND. Recognition of the ethical dilemmas faced by professionals involved in the PND service forms an important contribution to evaluating the practice of offering PND for AOCS. Some sense that the ethical debate about offering PND for AOCS has happened at some level before ending up as an option on the NHS website would be helpful.

\section{Acknowledgments}

We should like to acknowledge the HCPs who participated in this study and the Newcastle NHS Foundation Hospitals Trust who acted as sponsor.

\section{Author Contributions}

Lorraine Cowley was PI and supervisor on the project and co-analysed transcripts.

Mari Jones was student researcher on the project: interviewed participants, transcribed data and co-analysed transcripts.

\section{Funding}

This research was unfunded. It was submitted as a thesis for the MSc in Genomic Counselling (The University of Manchester) as part of the NHS Scientific Training Programme (STP) Health Education England (HEE) leading to professional qualification with the Health and Care Professions Council (HCPC).

\section{Competing Interests}

The authors have declared that no competing interests exist.

\section{References}

1. UKGOV. Abortion Act. Wales: legislation.gov.uk; 1967.

2. DOH. Form HSA1: Grounds for carrying out an abortion. Wales: GOV.UK; 2013.

3. Hunt K, Gruszczynski M. The ratification of CEDAW and the liberalization of abortion laws. Polit Gend. 2019; 15: 722-745.

4. Levels $M$, Sluiter $R$, Need A. A review of abortion laws in Western-European countries. A cross-national comparison of legal developments between 1960 and 2010. Health Pol. 2014; 118: 95-104.

5. Department of Health and Social Care. Abortion notification form for England and Wales HSA1. Wales: Gov.UK; 2013. pp. 1.

6. Choices, NHS. Predictive genetic tests for cancer risk genes. 2018. Available from: https://www.nhs.uk/conditions/predictive-genetic-tests-cancer/

7. NHS. Making the NHS website better for everyone. 2018. Available from: https://digital.nhs.uk/blog/transformation-blog/2018/making-the-nhs-website-better-foreveryone

8. The Royal Marsden NHS Foundation Trust. A beginner's guide to BRCA1 and BRCA2. 2016. Available from: https://shared-d7-royalmarsden-public.s3-eu-west1.amazonaws.com/files trust/s3fs-public/BRCA1 BRCA2 guide.pdf 
9. Staton $A D$, Kurian $A W$, Cobb K, Mills MA, Ford JM. Cancer risk reduction and reproductive concerns in female BRCA1/2 mutation carriers. Familial Cancer. 2007; 7: 179-186.

10. Quinn GV, Vadaparampil S, Wilson C, King L, Choi J, Miree C, et al. Attitudes of high-risk women toward preimplantation genetic diagnosis. Fertil Steril. 2009; 91: 2361-2368.

11. Ormondroyd E, Donnelly L, Moynihan C, Savona C, Bancroft E, Evans DG, et al. Attitudes to reproductive genetic testing in women who had a positive BRCA test before having children: A qualitative analysis. Eur J Human Genet. 2011; 20: 4-10.

12. van der Schoot V, Dondorp W, Dreesen JCFM, Coonen E, Paulussen ADC, de Wert G, et al. Preimplantation genetic testing for more than one genetic condition: Clinical and ethical considerations and dilemmas. Human Reprod. 2019; 34: 1146-1154.

13. Bayefsky M. Comparative preimplantation genetic diagnosis policy in Europe and the USA and its implications for reproductive tourism. Reprod Med Soc Online. 2016; 3: 41-47.

14. NHS England Clinical Reference Group for Medical Genetics. Pre-implantation Genetic Diagnosis (PGD) Clinical Commissioning Policy. 2014. E01/P/a.

15. Leonard A. Minutes of HFEA Meetings Granting PGD Licenses for BRCA1 and BRCA2. L. Cowley: HFEA; 2019.

16. Williams C, Ehrich K, Farsides B, Scott R. Facilitating choice, framing choice: Staff views on widening the scope of preimplantation genetic diagnosis in the UK. Soc Sci Med. 2007; 65: 1094-1105.

17. Wertz DC, Knoppers BM. Serious genetic disorders: Can or should they be defined? Amer J Med Genet. 2002; 108: 29-35.

18. Firth HV, Lindenbaum RH. UK clinicians' knowledge of and attitudes to the prenatal diagnosis of single gene disorders. J Med Genet. 1992; 29: 20-23.

19. Julian-Reynier C, Chabal F, Frebourg T, Lemery D, Noguès C, Puech F, et al. Professionals assess the acceptability of preimplantation genetic diagnosis and prenatal diagnosis for managing inherited predisposition to cancer. J Clin Oncol. 2009; 27: 4475-4480.

20. Williams $C$, Alderson $P$, Farsides B. Drawing the line' in prenatal screening and testing: Health practitioners' discussions. Health Risk Soc. 2002; 4: 61-75.

21. Parker M. Ethical problems and genetics practice. Cambridge: Cambridge University Press; 2012.

22. AGNC. Code of Ethics for Genetic Counsellors. 2011. Available from: http://www.agnc.org.uk.

23. Brandt AC. Tschirgi ML, Ready KJ, Sun C, Darilek S, Hecht J, et al. Knowledge, attitudes, and clinical experience of physicians regarding preimplantation genetic diagnosis for hereditary cancer predisposition syndromes. Family Cancer. 2010; 9: 479-487.

24. Jamshed S. Qualitative research method-interviewing and observation. J Basic Clin Pharm. 2014; 5: 87.

25. Riessman C. Analysis of personal narratives. Handbook of interview research: Context and method. California: G. J and H. J. Thousand Oaks; 2002. Sage: 695-711.

26. Glaser B. The constant comparative method of qualitative analysis. Soc Probl. 1965; 12: 436445.

27. Goodley D. Researching life stories: Method, theory and analyses in a biographical age. Qual Res. 2005; 3.

28. Byrne A, Canavan J, Millar M. Participatory research and the voice-centred relational method of data analysis: is it worth it? Int J Soc Res Methodol. 2009; 12: 67-77. 
29. Torrance $\mathrm{H}$. Triangulation, respondent validation, and democratic participation in mixed methods research. J Mix Meth Res. 2012; 6: 111-123.

30. Kleinman A. Suffering, ethics, and the politics of moral life. Cult Med Psych. 1996; 20: 287-290.

31. Plummer K. The call of life stories in ethnographic research. Handbook of Ethnography. London; 2001. Sage: 395-406.

32. Sayers $A$. Why things matter to people. social science, values and ethical life. Cambridge: Cambridge University Press; 2011.

33. Cowley L. What can we Learn from Patients' Ethical Thinking about the right 'not to know' in Genomics? Lessons from Cancer Genetic Testing for Genetic Counselling. Bioethics. 2016; 30: 628-635.

34. Czarnecki D, Anspach RR, De Vries RG, Dunn MD, Hauschildt K, Harris LH. Conscience reconsidered: The moral work of navigating participation in abortion care on labor and delivery. Soc Sci Med. 2019; 232: 181-189.

35. HFEA. Standing Orders, Annex A. 9.3 Particular requirements for applications authorising embryo testing. 2018. Available from: https://www.hfea.gov.uk/media/2555/hfea-standingorders-2018.pdf.

36. Evans MI, Drugan A, Bottoms SF, Platt L, Rodeck C, Hansmann M, et al. Attitudes on the ethics of abortion, sex selection and selective pregnancy termination among Health Care Professionals, Ethicists and Clergy likely to encounter such situations. Amer J Obstetr Gynaecol. 1991; 164: 1092-1099.

37. Hubbard R. Abortion and disability: Who should and who should not inhabit the world? The Disability Studies Reader. London: Routledge; 1997.

38. McLaughlin J. Screening Networks: Shared agendas in feminist and disability movement challenges to antenatal screening and abortion. Disabil Soc. 2003; 18: 297-310.

39. McMahan J. Paradoxes of abortion and prenatal injury. Ethics. 2006; 116: 625-655.

40. Halfmann D. Recognizing medicalization and demedicalization: Discourses, practices, and identities. Health. 2012; 16: 186-207.

41. Rollero MB. Discontinuation of pregnancy in obstetric practice: therapeutic resource versus provoked abortion. Acta Bioethica. 2016; 22: 169-178.

42. Chipman P. The moral implications of prenatal genetic testing. Penn Bioeth J. 2006; 2: 13-16.

43. Sirilla J. Moral distress in nurses providing direct care on inpatient oncology units. Clin J Oncol Nurs. 2014; 18: 536-541.

44. Robinson R, Stinson CK. Moral distress: A qualitative study of emergency nurses. Dimens Crit Care Nurs. 2016; 35: 235-240.

45. Gallagher J. Three-person babies - not three-parent babies. UK: BBC; 2015. Available from: https://www.bbc.co.uk/news/health-31044255.

46. Pal SK, Miller MJ, Agarwal N, Chang SM, Chavez-MacGregor M, Cohen E. Clinical Cancer Advances 2019- Annual Report on Progress against Cancer from the American Society of Clinical Oncology. J Clin Oncol. 2019; 37: 834-849.

47. NHS. UK Genetic Testing Network - Supporting Genetic Testing in the NHS. 2019. Available from: https://ukgtn.nhs.uk/

48. CRUK. PARP inhibitors. 2017. Available from: https://www.cancerresearchuk.org/aboutcancer/cancer-in-general/treatment/targeted-cancer-drugs/types/PARP-inhibitor. 
49. Snape K, Wedderburn S, Barwell J. The new genomic medicine service and implications for patients. Clin Med (Lond). 2019; 19: 273-277.

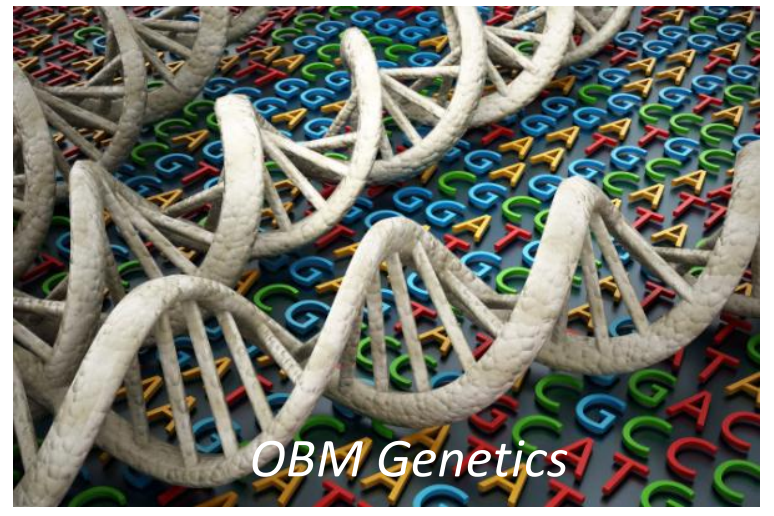

Enjoy OBM Genetics by:

1. Submitting a manuscript

2. Joining in volunteer reviewer bank

3. Joining Editorial Board

4. Guest editing a special issue

For more details, please visit:

http://www.lidsen.com/journals/genetics 